\title{
Harvesting the Mushroom Crop: a Mathematical Model
}

\author{
By D. O. CHANTER \\ Glasshouse Crops Research Institute, Littlehampton, West Sussex BN16 3PU
}

(Received 21 February 1979)

\begin{abstract}
A recently published mathematical model for mycelial growth and the initiation and growth of sporophores in the mushroom crop is extended to allow for harvesting of the sporophores. The extended model exhibits temporal fluctuations in yield which are similar, in most respects, to those produced by real crops. The response of the model to changes in the age at which sporophores are harvested is also similar to that observed experimentally, except that little change in total yield is predicted. It is suggested that this discrepancy is associated with the abortion of sporophore primordia, which is not allowed for in the model. The model is used to demonstrate the importance of using regular harvesting schedules in mushroom cropping experiments.
\end{abstract}

\section{INTRODUCTION}

Chanter \& Thornley (1978) described a mathematical model for a mushroom crop and gave a numerical solution up to the end of the first flush of sporophores for a particular set of parameter values. In this paper, the model is extended to include harvesting, and this enables the solution to be extended for several flushes.

The mathematical details of the basic model are not repeated in this paper, but a brief description follows. The model is a single-substrate three-compartment model, in which the substrate is an undefined carbon compound and the three compartments represent compost, mycelium and sporophores. The compartment representing mycelium is partitioned into structure and storage. The model applies from the time of spawning, and the following assumptions are made.

(i) The compost is homogeneous and contains a supply of substrate which acts as a source only. There is no input of substrate into the compost.

(ii) Substrate passes from the compost to the storage component of the mycelium at a rate which is proportional to the dry matter content of the structural component of the mycelium and to the difference between the substrate density in the compost and that in the mycelium.

(iii) Growth of the structural component of the mycelium is limited by an arbitrary upper asymptote. The specific growth rate of the mycelium is proportional to its substrate density and to the difference between its asymptotic and actual dry weights.

(iv) Initiation of sporophores occurs when (and only when) the substrate density in the mycelium exceeds a threshold level. The rate of initiation is proportional to the dry weight of the structural component of the mycelium and to the difference between the actual and threshold substrate densities in the mycelium.

(v) The specific growth rate of standing sporophores is proportional to an hyperbolic function of the substrate density in the mycelium and to a negative-exponential function of sporophore age.

(vi) Substrate requirements for growth of both the mycelium and the sporophores are met entirely by the substrate in the storage component of the mycelium. Energy for growth and maintenance is also provided by respiring substrate from this source. 
The model has 16 parameters, and the three principal state variables are $w_{m}$ (dry weight of mycelium), $s_{c}$ (substrate density in compost) and $s_{m}$ (substrate density in mycelium). The distribution of the number of sporophores, $n\left(t, t^{\prime}\right)$ [where $n\left(t, t^{\prime}\right) \mathrm{d} t^{\prime}$ is the number of sporophores at time $t$ which were initiated between times $t^{\prime}$ and $\left.t^{\prime}+\mathrm{d} t^{\prime}\right]$, and of the weight of sporophores, $w\left(t, t^{\prime}\right)$ (the weight at time $t$ of a sporophore initiated at time $t^{\prime}$ ), are also state variables.

The solution given previously (Chanter \& Thornley, 1978) terminated at the end of the first flush, when the sporophores had reached maturity. The addition of harvesting to the model enables the substrate density in the mycelium to build up again, and a second flush is produced.

\section{HARVESTING}

In practice, harvesting is usually based on the stage of development reached by the sporophores. Thus a crop is harvested as 'buttons' or 'cups' etc. In the model referred to above, the stage of development reached by a sporophore is not distinguished from its dry weight. The simplest assumption that can be made to overcome this difficulty is to assume that stage of development is a function of age only and not of dry weight. Harvesting by stage of development can then be incorporated into the model as harvesting by age.

The extension of the mathematical model to incorporate harvesting by age is quite straightforward. A parameter representing 'sporophore age at harvesting', $t_{h}$, is defined, and all sporophores are harvested as soon as they reach this age. The total number of sporophores existing at time $t, N(t)$, can then be written

$$
N(t)=N_{h}(t)+N_{s}(t)
$$

where $N_{h}(t)$ is the number which have been harvested by time $t$ and $N_{s}(t)$ is the number still standing at time $t$. The total weight of sporophores, $W(t)$, can be similarly subdivided. Formulae relating these quantities to the state variables of the model are given by Chanter (1976).

In practice, of course, it is not possible to harvest sporophores at the precise moment they reach a particular age. Usually the most that can be done is to examine the sporophores daily and harvest any which exceed a certain stage of development. The discrete nature of this process makes it difficult to incorporate it into the continuous formulation of the model given by Chanter \& Thornley (1978), but since the solution of the model is obtained by using a discrete version of this formulation, it is not difficult to apply harvesting during the computation of the solution. In addition to $t_{h}$, the age a sporophore must reach before it can be harvested, another parameter representing the time of day at which the harvesting examination is made must be defined. This latter parameter has only a small effect on the solution and is fixed at an arbitrary value in the results described here.

In this version of the model, then, sporophores are harvested when their age lies somewhere in the range $t_{h}$ to $t_{h}+1(\mathrm{~d})$.

\section{SOLUTION}

Using the parameter values given by Chanter \& Thornley (1978), a value of $t_{h}=4.58 \mathrm{~d}$ was found to give a solution in which the timing of the flushes is similar to that observed in practice. In this solution, four flushes are produced; after the fourth flush there is not enough substrate left in the compost to support any further initiation. Plots of the state variables $s_{c}, s_{m}$ and $w_{m}$ against $t$ are shown in Fig. 1 . The substrate density in the mycelium, $s_{m}$, reaches its maximum value during the initiation of the second flush; this is because less growth of the structural component of the mycelium is taking place during the initiation of the second flush than takes place during the initiation of the first.

A summary of the timing and yield of each flush is given in Table 1. The maximum 


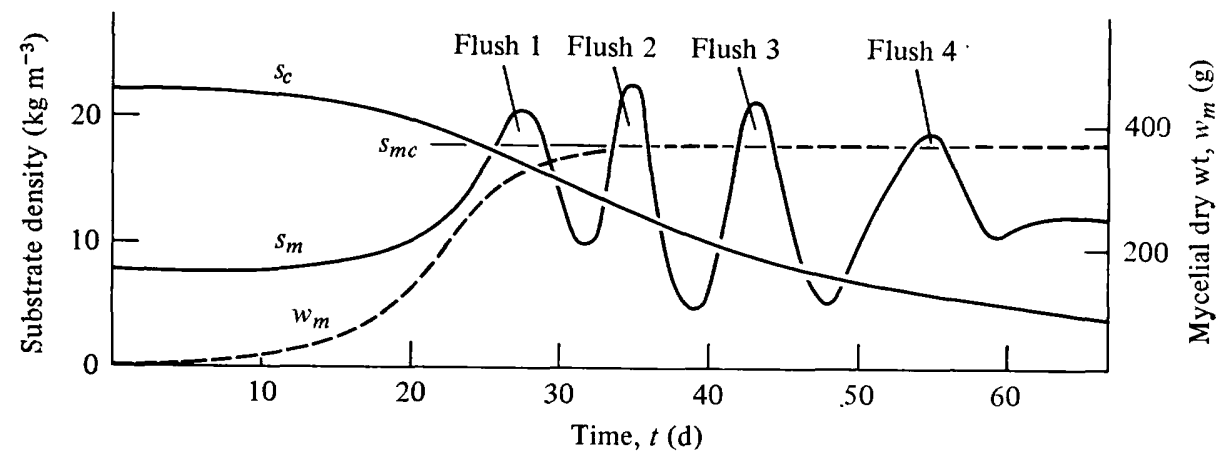

Fig. 1. Changes in the substrate densities in the compost and mycelium, $s_{c}$ and $s_{m}$ respectively ( - ), with time for the parameter values given by Chanter \& Thornley (1978) and with $t_{h}=4.6 \mathrm{~d}$. The critical substrate density in the mycelium, $s_{m c}$, and the dry weight of the mycelium, $w_{m}(---)$, are also shown.

Table 1. Summary of the timing and yield of the four flushes produced in the solution to the model with harvesting, using the parameter values given by Chanter \& Thornley (1978) with $t_{h}=4.58 d$, and (in italics) comparative data from the first four flushes produced by a typical crop grown at the Glasshouse Crops Research Institute

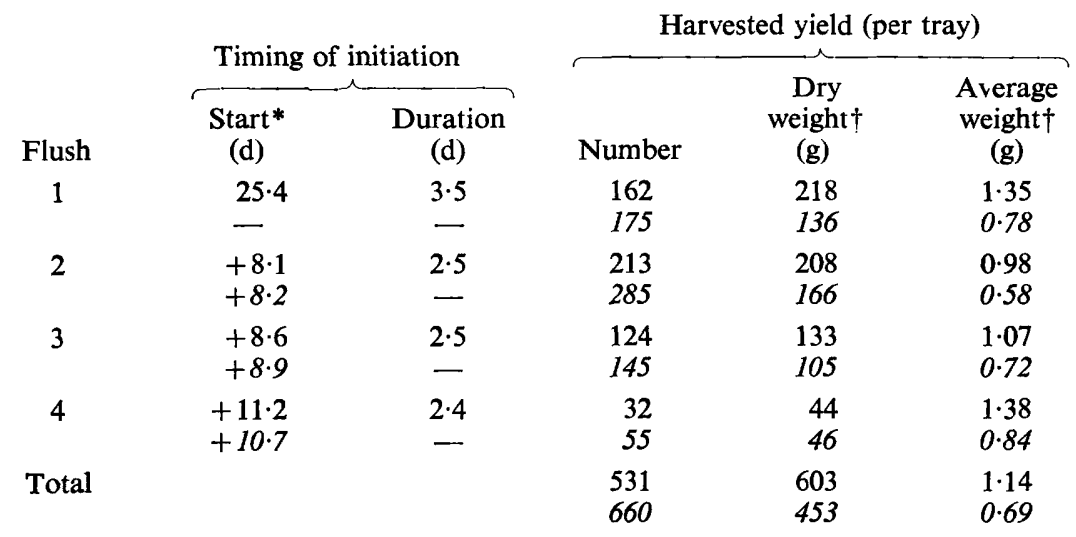

* The use of a plus sign in this column indicates that the value given is the difference between the time of initiation for the flush in question and that for the previous one. An arbitrary origin was used for the scale for the real data.

$\dagger$ For the real data, fresh marketable weights were measured and converted to dry weights by assuming a dry matter content of $9 \%$. No allowance was made for the portion of the stipe removed when preparing the sporophores for market.

number of sporophores initiated occurs in the second flush; this is commonly observed in practice and follows from the behaviour of $s_{m}(t)$ described above. Published data on mushroom crops usually contain information on yield but often not on numbers of sporophores, so comparison of these results with previously published data is not easy. Numbers of sporophores are sometimes recorded on experimental crops grown at the Glasshouse Crops Research Institute, and the data for one such crop are included in Table 1 . The experimental data on timing are not strictly comparable, because for the model the time at which initiation begins is used, whereas for the data the mean harvesting time is used. However, these quantities are closely correlated.

Apart from the fact that a value of $t_{h}=4.58 \mathrm{~d}$ was chosen to give the good agreement seen in Table 1 for the time interval between flushes, no attempt has been made to obtain as good a fit as possible for the number and weight of sporophores. Thus the fact that the 
Table 2. Distribution of substrate at time $t=0$ and after the final harvest of the first, second, third and fourth flushes $(t=35,42,51$ and $62 d)$ in the solution to the model with harvesting, using the parameter values given by Chanter \& Thornley (1978) and $t_{h}=4.58 d$

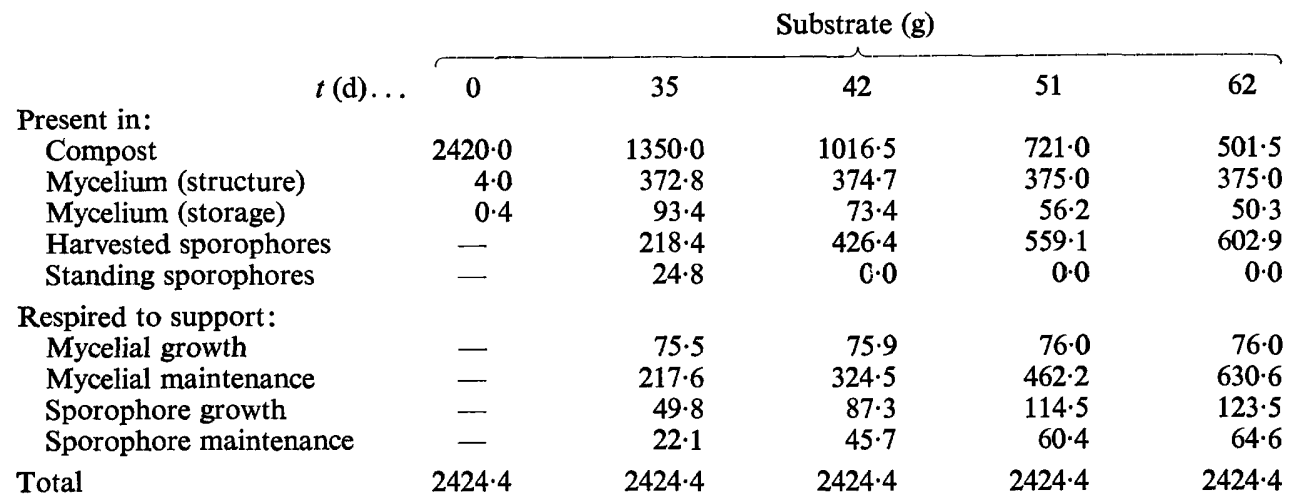

absolute values for these variates do not match closely is not serious. The changes in yield from flush to flush follow similar patterns in both the model and the real data.

Table 2 shows the distribution of substrate at various times during the course of the solution. By the end of the fourth flush, the dominant usages of substrate are mycelial maintenance and sporophore dry weight.

\section{SENSITIVITY ANALYSIS}

Chanter \& Thornley (1978) gave a sensitivity analysis of the model without harvesting, using outcomes such as the duration of initiation and the number of sporophores initiated. The extension of the model to include harvesting enables the range of outcomes investigated to be extended to include the total yield and the time interval between flushes. The effect of the new parameter $t_{h}$ is excluded from these analyses and is considered in the next section.

Increments of $10 \%$ were made to each parameter value in turn, and the effect on the solution was observed. The parameter having the biggest effect on total yield was the initial value of $s_{c}$, the substrate level in the compost; a $10 \%$ increment in this parameter resulted in a $25 \%$ increase in yield. This is because the requirements of the mycelium remain unchanged, and nearly all the extra substrate is available for sporophore production. No other parameter increase produces a change in excess of $10 \%$; details of these effects are given by Chanter (1976).

An investigation of the parameters affecting the time interval between the first and second flushes showed that parameter values had very little effect on this outcome, the maximum effect being less than $2 \%$. The generally small effect of parameter values on this time interval suggests that the timing of flushes in real crops should be relatively stable with regard to environmental conditions which may affect parameter values; this accords with the observations that flushing in mushroom crops is usually well synchronized.

\section{SPOROPHORE AGE AT HARVEST}

The incorporation of the parameter $t_{h}$, sporophore age at harvest, into the model provides a means by which predictions made by the model may be compared with published data.

Figure 2 shows the predicted effect of varying $t_{h}$ between about 3 and $8 \mathrm{~d}$ on the number, weight and average weight of sporophores harvested. With increasing $t_{h}$, the number decreases and the average weight increases in such a way that the total yield remains roughly 

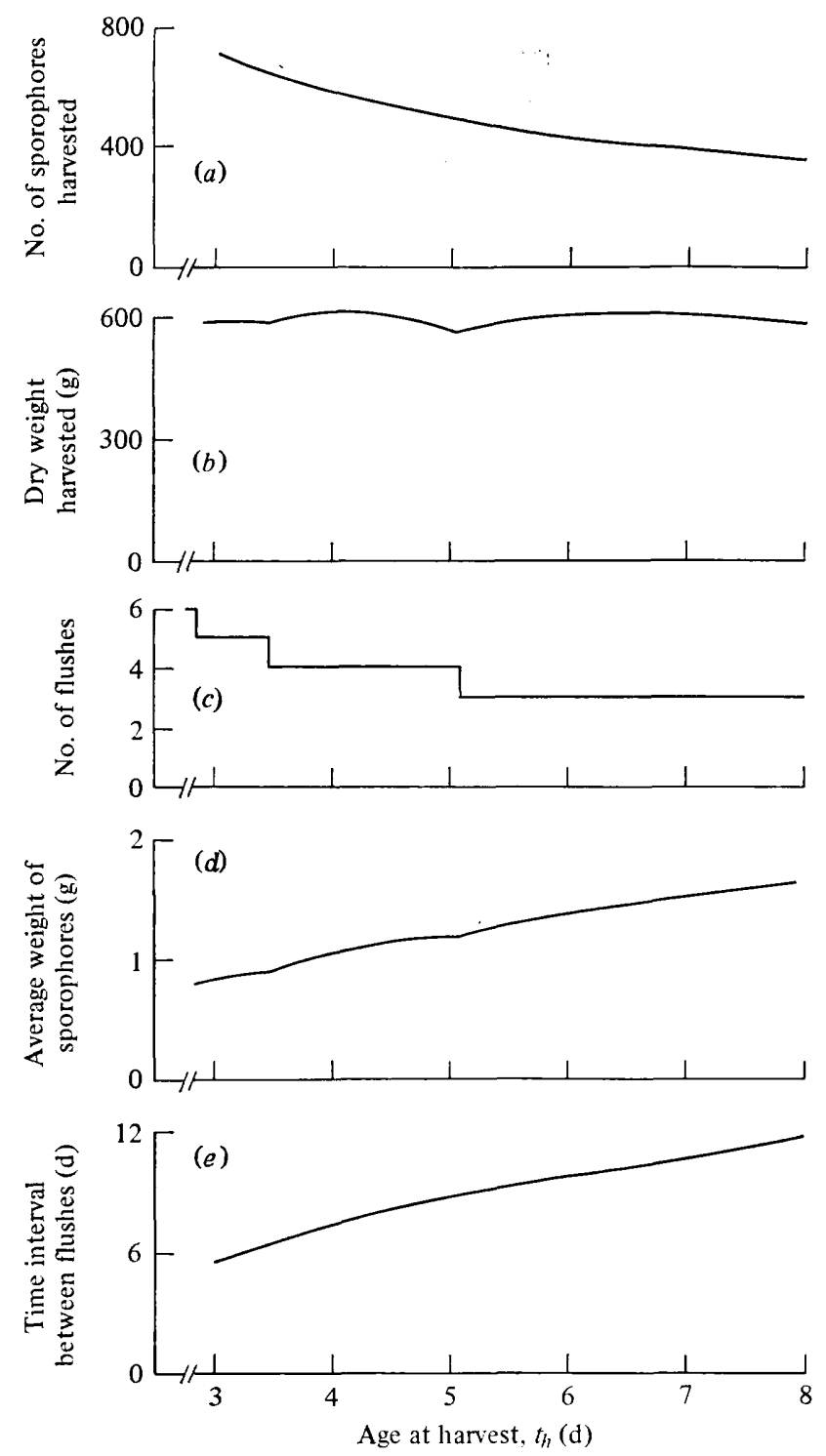

Fig. 2. Graphs showing the effect of the age at harvest, $t_{h}$, on various aspects of the solution to the model with harvesting, using the parameter values given by Chanter \& Thornley (1978). The graphs show $(a)$ the total number of sporophores harvested, $(b)$ the total dry weight of harvested sporophores, $(c)$ the number of flushes produced, $(d)$ the average dry weight of sporophores at harvest and $(e)$ the time interval between the beginning of initiation of the first and second flushes.

constant. Increasing $t_{h}$ also increases the time interval between the initiation of consecutive flushes; this effect for the interval between the first and second flushes is also shown in Fig. 2.

When $t_{h}$ is reduced below $2.5 \mathrm{~d}$, harvesting of the first flush begins before initiation has ceased. Thus the substrate requirement of the standing sporophores is reduced and the first flush is able to go on initiating longer. A reduction in $t_{h}$ from 2.50 to $2.08 \mathrm{~d}$ increases the duration of initiation from 3.5 to $7 \cdot 5 \mathrm{~d}$ and the number of sporophores initiated in the first flush from 162.7 to 384.7 (an increase of $136 \%$ ). The onset of the second flush is delayed by $4.1 \mathrm{~d}$. In later flushes the duration of initiation is shorter and is not affected by reducing $t_{h}$ to $2.08 \mathrm{~d}$. 
Most of the responses to changing $t_{h}$ which are predicted by the model have also been observed with real crops. Experiments in which the stage of maturity at harvest has been varied have been carried out by Edwards (1953), Rasmussen (1959), Kneebone \& Mason (1962) and Cooke \& Flegg $(1962,1965)$. The results of Cooke \& Flegg (1962) are typical, and the main conclusions are that as the stage of maturity at harvest is increased, the total number of sporophores harvested decreases, while increases are observed in the total yield, average yield and time between flushes. Thus the trends observed in real crops are in agreement with those predicted by the model with the exception of the increase in total yield in real crops. Numerical comparisons with the published data must be tentative, because the treatments are defined in terms of stage of maturity rather than sporophore age, but as the stage of maturity at picking was increased from buttons to flats the yield from 10 weeks' picking in Cooke \& Flegg's (1962) experiments increased by $58 \%$ (average for three crops). In the model, scarcely any change occurs (Fig. 2).

A possible reason for this discrepancy between the experimental results and the model may lie in the way in which initiation is modelled. The initiation of a sporophore in the model is assumed to require an amount of substrate equivalent to its initial dry weight, but in practice many primordia do not develop into mature sporophores, and these are ignored by the model. Cooke \& Flegg (1965) estimate the proportion of pinheads surviving to maturity at $56 \%$, but figures given by Flegg (1979) for the number of mycelial aggregates observed, taking into account those developing beneath the surface of the casing, suggest that the proportion of those which eventually develop into sporophores is far smaller, probably less than $1 \%$. The primordia which fail to develop presumably use some substrate, and for each sporophore which survives a substantial amount of substrate may be lost to primordia which abort. If this is the case, and the proportion of primordia surviving is constant, the increase in the number of sporophores initiated as $t_{h}$ is reduced, correctly predicted by the model, would imply a loss of more substrate to the aborted primordia, and hence the predicted total yield would fall.

Cooke \& Flegg (1965) made detailed observations on the duration of initiation (which they call the length of an 'appearance flush') and on the interval between flushes, as affected by the stage of maturity at harvest. In trays harvested as buttons, picking began before initiation had ceased, and initiation of the first flush in this treatment lasted $7.0 \mathrm{~d}$ compared with a mean of $4.3 \mathrm{~d}$ for the other treatments, but the duration of initiation of later flushes was not affected. The number of mushrooms in the first flush was also $116 \%$ larger than the mean for the other treatments. These observations compare well with the predictions based on the model when $t_{h}$ is reduced from 2.50 to $2.08 \mathrm{~d}$, which were described above. The predicted delay in the onset of the second flush was not observed.

The behaviour of the model when $t_{h}$ is changed therefore mimics real mushroom crops reasonably well, except with respect to the total yield, and better agreement in this respect could be obtained by introducing a further parameter into the model to allow for the abortion of some sporophore primordia.

\section{HARVESTING STRATEGIES IN CROPPING EXPERIMENTS}

Mushroom cropping experiments are expensive to carry out. Labour costs are high, and because the background variability is often high also (Chanter \& Flegg, 1973), the experiments have to be conducted on a large scale. Consequently, ways of reducing the cost are always being sought, and one economy often used in practice is to harvest the trays on weekdays only, and leave them unpicked on Saturdays and Sundays. When this is done, the Friday harvest is usually a 'hard pick', i.e. sporophores which would normally be left until the Saturday or Sunday are included in the Fiday harvest. This practice is contrary to the recommendation of Cooke \& Flegg (1963) that daily harvesting should be used in mushroom cropping experiments. 
Table 3. Effect of the phase of an irregular harvesting strategy on various responses, as predicted by the model, using the parameter values given by Chanter \& Thornley (1978)

The age at harvest, $t_{h}(\mathrm{~d})$, takes the sequence of values $(4 \cdot 58,10,4 \cdot 58,10,3.58,10,10)$, but the phase of the sequence is shifted one place (day) in successive solutions, so that the 'hard pick' falls on different days. The $t_{h}=10$ values correspond to no harvest on that day.

$\begin{array}{lccccc}\text { Weight } & \text { Total yield } & \begin{array}{c}\text { Average } \\ \text { weight } \\ (\mathrm{g})\end{array} & \begin{array}{c}\text { Weight in } \\ \text { first flush } \\ (\mathrm{g})\end{array} & \begin{array}{c}\text { Interval } \\ \text { between 1st } \\ \text { and 2nd } \\ \text { flushes } \\ \text { (d) }\end{array} \\ \text { Mean } & \begin{array}{c}\text { Number } \\ \text { Minimum }\end{array} & 593 & 1 \cdot 19 & 231 & 8.51 \\ \text { Maximum } & 500 & 578 & 1 \cdot 14 & 211 & 7 \cdot 66 \\ \text { Percentage difference } & 475 & 609 & 1.22 & 239 & 8.83\end{array}$

between max. and

$\min$.

In view of the fact that harvesting is known to have a marked effect on the timing of flushes, it seems likely that the use of this strategy will introduce a bias into the estimation of treatment means in an experiment in which the treatments affect timing. This is because the harvesting strategy will tend to synchronize the flushing patterns of different treatments.

An experimental investigation of the bias produced by this harvesting strategy could be done by growing seven crops simultaneously, each one started on a different day of the week. An easier method is to grow one crop, but to apply seven different harvesting treatments; one treatment is harvested on Monday to Friday (with a hard pick on Friday), the next is harvested on Tuesday to Saturday, etc. The maximum treatment differences encountered would indicate the maximum size of bias which could be introduced if such a harvesting strategy were to be used in an experiment in which the treatments affect the timing of the crop.

A harvesting experiment of the kind described above can be simulated using the model. A 'weekdays only' harvesting strategy can be represented by allowing $t_{h}$ to take a repeating sequence of values such as $4 \cdot 58,4 \cdot 58,4 \cdot 58,4 \cdot 58,3 \cdot 58, *, * \mathrm{~d}$. The smaller value of $t_{h}(3 \cdot 58 \mathrm{~d})$ is chosen to represent a hard pick, but could probably be made even smaller without being unrealistic. An asterisk $\left(^{*}\right)$ denotes no harvest; this can be achieved by setting $t_{h}$ to a value greater than the maximum attainable. Seven different solutions can be obtained, with the cycle of $t_{h}$ values starting at different days in the sequence.

This has been done using a slightly modified sequence designed to represent a situation in which further economies are sought by picking on only three out of the five weekdays; the actual sequence used was $4 \cdot 58, *, 4 \cdot 58, *, 3 \cdot 58, *, *$. The results are summarized in Table 3 ; the maximum differences between solutions range from $5.3 \%$ for the total yield to $15.3 \%$ for the interval between the first and second flushes. Since, as described above, the total yield was more affected by harvesting strategy in reality than in the model, the value of $5.3 \%$ obtained for the total yield could be an underestimate of the maximum amount of bias that irregular harvesting strategies can introduce. The effect on the time interval between flushes is also large; $15 \cdot 3 \%$ represents a change of more than $1 \mathrm{~d}$ in the timing of the second flush, and in experiments designed to study timing such a bias would be intolerable.

\section{DISCUSSION}

The addition of harvesting to the model described by Chanter \& Thornley (1978) makes it easier to compare the behaviour of the model with that of real crops. The most conspicuous point of similarity is the fact that flushes are produced, but in addition to this there are many points of similarity in the pattern of flushing. These include: (i) The number of sporophores 
produced in each flush can have a maximum value in either the first or the second flush, and after this the number becomes progressively smaller. (ii) The more mushrooms initiated in a flush, the smaller their average weight tends to be. (iii) During the initiation of a flush, the rate of initiation rises to a maximum and then decreases. (iv) Mushrooms initiated at the beginning of a flush grow more quickly than those initiated at the end. (v) If mushrooms from a given flush are harvested while that flush is still initiating, the duration of the flush and the number of sporophores initiated are both increased. (vi) If sporophores are harvested at a later stage of maturity, flushes appear further apart in time, and the total number of sporophores harvested is reduced. (vii) The duration of initiation of the first flush is longer than that of subsequent flushes.

These similarities are sufficient to indicate that the model might have some potential as a research tool, particularly if it were used in a modified form taking account of the abortion of sporophores. One application of the model, the investigation of harvesting strategies in cropping experiments, has already been discussed. Another possible use is in the investigation of the effect of insecticide and fungicide applications on the cropping pattern and on yield. The effect of insecticides has been investigated experimentally by Wyatt (1978) and typical response patterns have been quantified. Hypotheses about the mode of action of the insecticides could be built into the model to see whether the response is similar to that obtained in practice.

Before the model could be of much use as a management tool, it would be necessary to determine the effects of environmental and cultural operations on the parameters of the model; this is likely to be difficult, as it is not possible to measure most of the parameters. However, some information useful in management can be obtained from the model as it stands. For example, the model predicts that any parameter change which results in an earlier first flush will also reduce the time interval between the first and second flushes, and the second flush will therefore be advanced further than the first. If, therefore, a grower wishes to speed up his first flush but leave his second unaffected, he would have to take positive steps to delay the second flush.

Whilst it is hoped that the model will be of use as a research tool, it is clearly oversimplified, and some of the assumptions on which it is based are not supported by experimental evidence. Therefore, any conclusions derived from the model would need to be backed up by experimental work. One of the major assumptions of the model concerns the way in which the cropping behaviour is related to the substrate level, $s_{c}$. Recent experimental work (Hammond \& Nichols, 1979) has shown that two of the non-structural carbohydrates present in the mushroom, trehalose and glycogen, do show fluctuations similar to those shown by $s_{c}$ in Fig. 1 . To some extent this strengthens the assumptions made in the model, although further refinements of this aspect of the model will not be possible until the functions of these carbohydrates and of the most abundant soluble carbohydrate, mannitol, are better understood.

\section{REFERENCES}

ChANTER, D. O. (1976). Mathematical models in mushroom research and production. D.Phil. thesis, University of Sussex.

Chanter, D. O. \& Flegg, P. B. (1973). Bulk recording of mushrooms from randomised block experiments. Annual Report, Glasshouse Crops Research Institute 1972, 111-115.

Chanter, D. O. \& Thornley, J. H. M. (1978). Mycelial growth and the initiation and growth of sporophores in the mushroom crop: a mathematical model. Journal of General Microbiology 106, 55-65.

CoOKe, D. \& FlegG, P. B. (1962). The relation between yield of the cultivated mushroom and the stage of maturity at picking. Journal of Horticultural Science 37, 167-174.

CoOKe, D. \& FlegG, P. B. (1963). The conduct of yield experiments with mushrooms. Mushroom Science V, 425-436.

COOKe, D. \& FleGG, P. B. (1965). The effect of stage of maturity at picking on the flushing of crops of the cultivated mushroom. Journal of Horticultural Science 40, 207-212.

EDWARDS, R. L. (1953). General cropping experiments. Annual Report, Mushroom Research Station, Yaxley, 1952, 30-37. 
FlegG, P. B. (1979). Effect of temperature on sporophore initiation and development in Agaricus bisporus. Mushroom Science (in the Press).

Hammond, J. B. W. \& Nichols, R. (1979). Carbohydrate metabolism in Agaricus bisporus: changes in non-structural carbohydrates during periodic fruiting (flushing). New Phytologist (in the Press). KNEEbone, L. R. \& Mason, E. C. (1962). Mushroom yields as influenced by degree of maturity at time of harvest. Mushroom Science V, 448-453.
RASMUSSEN, C. R. (1959). Plukkestorrelsens indflydelse paa hostudbyttet (Picking size in relation to cropping yield). 7th Report from the Danish Mushroom Research Station, 51-54.

WyatT, I. J. (1978). Principles of insecticide action on mushroom cropping: incorporation into casing. Annals of Applied Biology 88, 89-103 